\title{
The Fock-Rosly Poisson Structure as Defined by a Quasi-Triangular $r$-Matrix
}

Victor MOUQUIN

University of Toronto, Toronto ON, Canada

E-mail: mouquinv@math.toronto.edu

Received March 26, 2017, in final form August 01, 2017; Published online August 09, 2017 https://doi.org/10.3842/SIGMA.2017.063

\begin{abstract}
We reformulate the Poisson structure discovered by Fock and Rosly on moduli spaces of flat connections over marked surfaces in the framework of Poisson structures defined by Lie algebra actions and quasitriangular $r$-matrices, and we show that it is an example of a mixed product Poisson structure associated to pairs of Poisson actions, which were studied by J.-H. Lu and the author. The Fock-Rosly Poisson structure corresponds to the quasi-Poisson structure studied by Massuyeau, Turaev, Li-Bland, and Ševera under an equivalence of categories between Poisson and quasi-Poisson spaces.
\end{abstract}

Key words: flat connections; Poisson Lie groups; $r$-matrices; quasi-Poisson spaces

2010 Mathematics Subject Classification: 53D17; 53D30; 17B62

\section{Introduction}

Let $G$ be a connected complex Lie group with Lie algebra $\mathfrak{g}$, and let $s \in S^{2} \mathfrak{g}$ be a $\mathfrak{g}$-invariant element. The moduli space of flat $G$-connections on a Riemann surface $\Sigma$ has the well known [3] canonical Atiyah-Bott Poisson structure. If one "marks" finitely many points $V \subset \partial \Sigma$ in the boundary of $\Sigma$ and consider only gauge transformations which are trivial over $V$, Fock and Rosly have constructed in $[4,5]$ a Poisson structure $\pi_{r}$ on the corresponding moduli space $\mathcal{A}(\Sigma, V)$, which depends on a quasitriangular $r$-matrix $r_{v}$ for every $v \in V$ such that all $r_{v}$ 's have symmetric part $s$. Under the quotient by the group of lattice gauge transformations $G^{V}, \pi_{r}$ descends to the Atiyah-Bott Poisson structure on the full moduli space, and quantizations of $\pi_{r}$ play a fundamental role in quantum gravity (see [10] and references therein).

The bivector field $\pi_{r}$ was given in $[4,5]$ by a formula, of which the proof that it defines a Poisson structure was left as a computation. In this paper, as an application of the methods developed in [8], we give a simpler and more conceptual proof that $\pi_{r}$ is a Poisson structure, by viewing it in the framework of Poisson structures defined by a Lie algebra action and a quasitriangular $r$-matrix. Recall that given an action $\rho: \mathfrak{h} \rightarrow \mathfrak{X}^{1}(Y)$ of a Lie algebra $\mathfrak{h}$ on a manifold $Y$ and a quasitriangular $r$-matrix $r \in \mathfrak{h} \otimes \mathfrak{h}$, if the pushforward $\pi_{Y}=\rho(r)$ is a bivector field, it is automatically Poisson, and one says that $\pi_{Y}$ is defined by the action $\rho$ and the $r$-matrix $r$.

More precisely, given an oriented skeleton $\Gamma$ of a marked surface $(\Sigma, V)$, one has a natural action $\sigma_{\Gamma}$ of the Lie algebra $\mathfrak{g}^{\Gamma_{1 / 2}}$ on $\mathcal{A}(\Sigma, V)$, where $\Gamma_{1 / 2}$ is the set of half edges of $\Gamma$, and a quasitriangular $r$-matrix $r_{\Gamma} \in \mathfrak{g}^{\Gamma_{1 / 2}} \otimes \mathfrak{g}^{\Gamma_{1 / 2}}$, such that $\sigma_{\Gamma}\left(r_{\Gamma}\right)$ is a Poisson structure. Both $\sigma_{\Gamma}$ and $r_{\Gamma}$ depend on $\Gamma$, but one proves that $\pi_{r}=\sigma_{\Gamma}\left(r_{\Gamma}\right)$ does not.

Marked surfaces can be fused at their marked points. One also has the notion introduced in [8] of fusion of Poisson spaces admitting a Poisson action by a quasitriangular Lie bialgebra, and we show that the Poisson structures on the associated moduli spaces correspond under these constructions. In particular, the Fock-Rosly Poisson structure is an example of a mixed product Poisson structure associated to pairs of Poisson actions introduced in [8]. 
On the other hand, $\mathcal{A}(\Sigma, V)$ carries a canonical quasi-Poisson structure $Q_{s}$, first discovered in [9] when $V$ is a singleton, and further studied in [7] for general $V$ 's, which can be obtained by reduction of the canonical symplectic structure on the infinite-dimensional affine space of $G$ connections on $\Sigma$. Quasi-Poisson manifolds were introduced in [1,2] as a way to obtain a unified picture of various notions of moment maps. It is shown in $[1,6,8]$ (see also Section 5.1) that one has an equivalence of categories between the category of $\left(\mathfrak{g}, \phi_{s}\right)$ quasi-Poisson spaces and the category of $(\mathfrak{g}, r)$ Poisson spaces, where $r$ is a quasitriangular $r$-matrix whose symmetric part is $s$, and $\phi_{s} \in \wedge^{3} \mathfrak{g}$ is the Cartan element associated to $s$ (see (2.1)). We show in this paper that $\pi_{r}$ corresponds to $Q_{s}$ under this equivalence of categories.

An interesting project would be to develop a theory of quantizations of Poisson structures defined by actions of Lie algebras and quasitriangular $r$-matrices. This paper provides the setting to study the quantization of the Fock-Rosly Poisson structure from this point of view.

The paper is organized as follows. In Section 2 we recall the basic facts on quasitriangular $r$-matrices which will be needed later, and in Section 3 we recall the fusion of ciliated graphs and marked surfaces. The Poisson structure $\pi_{r}$ on the moduli space $\mathcal{A}(\Sigma, V)$ is defined in Section 4, where we prove that it is independent of the choice of an oriented skeleton of $(\Sigma, V)$, and that fusion of marked surfaces corresponds to fusion of the associated Poisson structures. In Section 5 , the equivalence between $\pi_{r}$ and the quasi-Poisson structure $Q_{s}$ under an equivalence of categories between Poisson and quasi-Poisson spaces is proven.

\section{$1.1 \quad$ Notation}

Throughout this paper, vector spaces are understood to be over $\mathbb{R}$ or $\mathbb{C}$.

If $\Gamma$ is a finite set and $\left\{X_{\gamma}: \gamma \in \Gamma\right\}$ a family of sets indexed by $\Gamma$, for $x \in \prod_{\gamma \in \Gamma} X_{\gamma}$ and $\gamma \in \Gamma, x_{\gamma} \in X_{\gamma}$ denotes the $\gamma$-component of $x$. If $\left\{V_{\gamma}: \gamma \in \Gamma\right\}$ is a family of groups and $v \in V_{\gamma}$, $(v)_{\gamma} \in \bigoplus_{\gamma \in \Gamma} V_{\gamma}$ is the image of $v$ under the embedding of $V_{\gamma}$ into $\bigoplus_{\gamma \in \Gamma} V_{\gamma}$ as the $\gamma$-component. When the $V_{\gamma}$ 's are vector spaces, we extend this notation to tensor powers. Namely, for an integer $k \geq 1$ and $v \in V_{\gamma}^{\otimes k},(v)_{\gamma}$ is the image of $v$ under the embedding of $V_{\gamma}^{\otimes k}$ into $\left(\bigoplus_{\gamma \in \Gamma} V_{\gamma}\right)^{\otimes k}$ as the $\gamma$-component.

If $\rho: Y \times G \rightarrow Y$ (resp. $\lambda: G \times Y \rightarrow Y$ ) is a right (resp. left) action of a Lie group $G$ on a manifold $Y$, we will denote by $\rho: \mathfrak{g} \rightarrow \mathfrak{X}^{1}(Y)$ (resp. $\lambda: \mathfrak{g} \rightarrow \mathfrak{X}^{1}(Y)$ ) the induced right (resp. left) Lie algebra action of the Lie algebra $\mathfrak{g}$ of $G$ on $Y$. If $x \in \mathfrak{g}^{\otimes k}, k \geq 1$, we denote respectively by $x^{R}$ and $x^{L}$ the right and left invariant $k$-tensor field on $G$ whose value at the identity $e \in G$ is $x$.

Lie bialgebras will be denoted as pairs $\left(\mathfrak{g}, \delta_{\mathfrak{g}}\right)$, where $\mathfrak{g}$ is a Lie algebra, and $\delta_{\mathfrak{g}}: \mathfrak{g} \rightarrow \wedge^{2} \mathfrak{g}$ the cocycle map. Recall that $\delta_{\mathfrak{g}}$ satisfies

$$
\delta_{\mathfrak{g}}([x, y])=\left[x, \delta_{\mathfrak{g}}(y)\right]+\left[\delta_{\mathfrak{g}}(x), y\right], \quad x, y \in \mathfrak{g},
$$

and that the dual map $\delta_{\mathfrak{g}}^{*}: \wedge^{2} \mathfrak{g}^{*} \rightarrow \mathfrak{g}^{*}$ is a Lie bracket on $\mathfrak{g}^{*}$.

\section{Poisson structures defined by quasitriangular $r$-matrices}

We recall in this section basic facts about quasitriangular $r$-matrices and refer to [8] for a detailed exposition on Poisson Lie groups and Lie bialgebras.

\subsection{Quasitriangular $r$-matrices}

Let $\mathfrak{g}$ be a finite-dimensional Lie algebra, and let $r=s+\Lambda \in \mathfrak{g} \otimes \mathfrak{g}$, with $s \in\left(S^{2} \mathfrak{g}\right)^{\mathfrak{g}}$ and $\Lambda \in \wedge^{2} \mathfrak{g}$. One says that $r$ is a quasitriangular $r$-matrix on $\mathfrak{g}$ if it satisfies the classical Yang-Baxter equation

$$
[\Lambda, \Lambda]+\phi_{s}=0
$$


where [, ]: $\wedge^{k} \mathfrak{g} \otimes \wedge^{l} \mathfrak{g} \rightarrow \wedge^{k+l-1} \mathfrak{g}$ is the Schouten bracket on the exterior powers of a Lie algebra, and $\phi_{s} \in \wedge^{3} \mathfrak{g}$ is defined by

$$
\phi_{s}(\xi, \eta, \zeta)=2\left\langle\xi,\left[s^{\#}(\eta), s^{\#}(\zeta)\right]\right\rangle, \quad \xi, \eta, \zeta \in \mathfrak{g}^{*},
$$

where $s^{\sharp}: \mathfrak{g}^{*} \rightarrow \mathfrak{g}$ is given by $\left\langle s^{\sharp}(\xi), \eta\right\rangle=s(\xi, \eta), \xi, \eta \in \mathfrak{g}^{*}$. If $r=\sum_{i} x_{i} \otimes y_{i} \in \mathfrak{g} \otimes \mathfrak{g}$ is a quasitriangular $r$-matrix, it defines a Lie bialgebra structure

$$
\delta_{r}: \mathfrak{g} \rightarrow \mathfrak{g} \wedge \mathfrak{g}, \quad \delta_{r}(x)=\sum_{i}\left[x, x_{i}\right] \otimes y_{i}+x_{i} \otimes\left[x, y_{i}\right], \quad x \in \mathfrak{g},
$$

and one calls the pair $(\mathfrak{g}, r)$ a quasitriangular Lie bialgebra.

Let $\left(\mathfrak{g}, \delta_{\mathfrak{g}}\right)$ be a Lie bialgebra and $\left(Y, \pi_{Y}\right)$ a Poisson manifold. A (right) Poisson action of $\left(\mathfrak{g}, \delta_{\mathfrak{g}}\right)$ on $\left(Y, \pi_{Y}\right)$ is a Lie algebra morphism $\rho: \mathfrak{g} \rightarrow \mathfrak{X}^{1}(Y)$ satisfying

$$
\left[\rho(x), \pi_{Y}\right]=\rho\left(\delta_{\mathfrak{g}}(x)\right), \quad x \in \mathfrak{g},
$$

and one also says that $\left(Y, \pi_{Y}, \rho\right)$ is a right $\left(\mathfrak{g}, \delta_{\mathfrak{g}}\right)$-Poisson space.

Let $\mathfrak{g}$ be a finite-dimensional Lie algebra, $Y$ a manifold and $\rho: \mathfrak{g} \rightarrow \mathfrak{X}^{1}(Y)$ a right action of $\mathfrak{g}$ on $Y$. For $r=\sum_{i} x_{i} \otimes y_{i} \in \mathfrak{g} \otimes \mathfrak{g}$, one has the 2 -tensor field

$$
\rho(r)=\sum_{i} \rho\left(x_{i}\right) \otimes \rho\left(y_{i}\right) \in \mathfrak{X}^{1}(Y) \otimes \mathfrak{X}^{1}(Y),
$$

and writing $r=s+\Lambda$, with $s \in S^{2} \mathfrak{g}$ and $\Lambda \in \wedge^{2} \mathfrak{g}$, it is clear that $\rho(r)$ is a bivector field on $Y$ if and only if $\rho(s)=0$.

Proposition 2.1 ([8, Proposition 2.18]). If $r$ is a quasitriangular $r$-matrix and if $\rho(r)$ is a bivector field, it is a Poisson bivector field, and $(Y, \rho(r), \rho)$ is a right $(\mathfrak{g}, r)$-Poisson space.

In the context of Proposition 2.1, one says that $\rho(r)$ is a Poisson structure defined by the quasitriangular $r$-matrix $r$ and the action $\rho$.

Let $\mathfrak{g}$ be a Lie algebra and $n \geq 1$ an integer. For any $r=\sum_{i} x_{i} \otimes y_{i} \in \mathfrak{g} \otimes \mathfrak{g}$, define $\operatorname{Mix}^{n}(r) \in \wedge^{2}\left(\mathfrak{g}^{n}\right)$ by

$$
\operatorname{Mix}^{n}(r)=\sum_{1 \leq j<k \leq n}\left(\operatorname{Mix}^{n}(r)\right)_{j, k},
$$

where

$$
\left(\operatorname{Mix}^{n}(r)\right)_{j, k}=\sum_{i}\left(y_{i}\right)_{j} \wedge\left(x_{i}\right)_{k}, \quad 1 \leq j<k \leq n,
$$

and for any sign function $\varepsilon:\{1, \ldots, n\} \rightarrow\{1,-1\}$, let

$$
r^{\varepsilon, n}=(\varepsilon(1) s, \ldots, \varepsilon(n) s)+(\Lambda, \ldots, \Lambda) \in \mathfrak{g}^{n} \otimes \mathfrak{g}^{n},
$$

where $r=s+\Lambda$ with $s \in S^{2} \mathfrak{g}$ and $\Lambda \in \wedge^{2} \mathfrak{g}$, and let

$$
r^{(\varepsilon, n)}=r^{\varepsilon, n}-\operatorname{Mix}^{n}(r) \in \mathfrak{g}^{n} \otimes \mathfrak{g}^{n} .
$$

Theorem 2.2 ([8, Theorem 6.2]). If $r \in \mathfrak{g} \otimes \mathfrak{g}$ is a quasitriangular r-matrix on $\mathfrak{g}$, then for any $n \geq 1$ and any sign function $\varepsilon, r^{(\varepsilon, n)}$ is a quasitriangular $r$-matrix on $\mathfrak{g}^{n}$, and the Lie bialgebra structure

$$
\delta_{r}^{(n)}=\delta_{r(\varepsilon, n)}
$$

is independent of $\varepsilon$. Moreover, the map

$$
\operatorname{diag}_{n}:\left(\mathfrak{g}, \delta_{r}\right) \rightarrow\left(\mathfrak{g}^{n}, \delta_{r}^{(n)}\right), \quad \operatorname{diag}_{n}(x)=(x, \ldots, x), \quad x \in \mathfrak{g},
$$

is an embedding of Lie bialgebras. 
For any $r \in \mathfrak{g} \otimes \mathfrak{g}$ and any sign function $\varepsilon$, denote by $\Lambda_{r}^{(n)}$ the anti-symmetric part of $r^{(\varepsilon, n)}$. Writing $r=\sum_{i} x_{i} \otimes y_{i}=s+\Lambda$, with $2 s=\sum_{i}\left(x_{i} \otimes y_{i}+y_{i} \otimes x_{i}\right)$ and $2 \Lambda=\sum_{i} x_{i} \wedge y_{i}$, one has explicitly

$$
\Lambda_{r}^{(n)}=(\Lambda, \ldots, \Lambda)-\operatorname{Mix}^{n}(r)=\frac{1}{2} \sum_{j=1}^{n} \sum_{i}\left(x_{i}\right)_{j} \wedge\left(y_{i}\right)_{j}-\sum_{1 \leq j<k \leq n} \sum_{i}\left(y_{i}\right)_{j} \wedge\left(x_{i}\right)_{k} .
$$

The following lemma will be used in the proof of Proposition 5.2.

Lemma 2.3. Let $r=s+\Lambda \in \mathfrak{g} \otimes \mathfrak{g}$, with $s \in S^{2} \mathfrak{g}$ and $\Lambda \in \wedge^{2} \mathfrak{g}$. Then

$$
\Lambda_{r}^{(n)}-\operatorname{diag}_{n}(\Lambda)=-\operatorname{Mix}^{n}(s) .
$$

Proof. A straightforward calculation shows that $\operatorname{diag}_{n}(\Lambda)=(\Lambda, \ldots, \Lambda)-\operatorname{Mix}^{n}(\Lambda)$. Thus

$$
\Lambda_{r}^{(n)}-\operatorname{diag}_{n}(\Lambda)=-\operatorname{Mix}^{n}(r)+\operatorname{Mix}^{n}(\Lambda)=-\operatorname{Mix}^{n}(s) .
$$

The following lemma will be used in the proof of Theorem 4.10.

Lemma 2.4. Let $r \in \mathfrak{g} \otimes \mathfrak{g}$. For integers $m, n \geq 0$, one has

$$
\left(\Lambda_{r}^{(m)}, \Lambda_{r}^{(n)}\right)-\left(\operatorname{diag}_{m}, \operatorname{diag}_{n}\right)\left(\operatorname{Mix}^{2}(r)\right)=\Lambda_{r}^{(m+n)} \in \wedge^{2}\left(\mathfrak{g}^{m+n}\right) .
$$

Proof. Indeed, writing $r=\sum_{i} x_{i} \otimes y_{i}$ and letting $\Lambda \in \wedge^{2} \mathfrak{g}$ be the anti-symmetric part of $r$, one has

$$
\left(\operatorname{diag}_{m}, \operatorname{diag}_{n}\right)\left(\operatorname{Mix}^{2}(r)\right)=\sum_{1 \leq k \leq m, 1 \leq l \leq n}\left(y_{i}\right)_{k} \wedge\left(x_{i}\right)_{l},
$$

hence

$$
\begin{aligned}
& \left(\Lambda_{r}^{(m)}, \Lambda_{r}^{(n)}\right)-\left(\operatorname{diag}_{m}, \operatorname{diag}_{n}\right)\left(\operatorname{Mix}^{2}(r)\right) \\
& \quad=(\Lambda, \ldots, \Lambda)-\sum_{1 \leq k<l \leq m+n}\left(y_{i}\right)_{k} \wedge\left(x_{i}\right)_{l}=\Lambda_{r}^{(m+n)} .
\end{aligned}
$$

\subsection{Fusion products of Poisson spaces}

Let $n \geq 1$ be an integer, $r \in \mathfrak{g} \otimes \mathfrak{g}$ a quasitriangular $r$-matrix on a Lie algebra $\mathfrak{g}$, and let $\left(Y, \pi_{Y}\right)$ be a Poisson manifold with a right Poisson action $\rho: \mathfrak{g}^{n} \rightarrow \mathfrak{X}^{1}(Y)$ of $(\mathfrak{g}, r)^{n}$, and a right Poisson action $\psi: \mathfrak{h} \rightarrow \mathfrak{X}^{1}(Y)$ of a Lie bialgebra $\left(\mathfrak{h}, \delta_{\mathfrak{h}}\right)$, so that $\left(Y, \pi_{Y}, \rho \times \psi\right)$ is a right $(\mathfrak{g}, r)^{n} \times\left(\mathfrak{h}, \delta_{\mathfrak{h}}\right)$ Poisson space. By [8, Lemma 2.16] and Theorem 2.2, the triple

$$
\operatorname{Fus}_{(\mathfrak{g}, r)^{n}}\left(Y, \pi_{Y}, \rho \times \psi\right):=\left(Y, \pi_{Y}-\rho\left(\operatorname{Mix}^{n}(r)\right),\left(\rho \circ \operatorname{diag}_{n}\right) \times \psi\right)
$$

is a right $(\mathfrak{g}, r) \times\left(\mathfrak{h}, \delta_{\mathfrak{h}}\right)$-Poisson space, which we call the fusion at $(\mathfrak{g}, r)^{n}$ of $\left(Y, \pi_{Y}, \rho \times \psi\right)$. As a particular case, suppose that $\mathfrak{h}=0$, that

$$
\left(Y_{1}, \pi_{Y_{1}}, \rho_{1}\right), \ldots,\left(Y_{n}, \pi_{Y_{n}}, \rho_{n}\right)
$$

are right $(\mathfrak{g}, r)$-Poisson spaces, that $Y=Y_{1} \times \cdots \times Y_{n}$ is equipped with the direct product Poisson structure $\pi_{Y}=\pi_{Y_{1}} \times \cdots \times \pi_{Y_{n}}$, and that $\rho: \mathfrak{g}^{n} \rightarrow \mathfrak{X}^{1}(Y)$ is given by

$$
\rho\left(x_{1}, \ldots, x_{n}\right)=\left(\rho_{1}\left(x_{1}\right), \ldots, \rho_{n}\left(x_{n}\right)\right), \quad x_{j} \in \mathfrak{g} .
$$

The $(\mathfrak{g}, r)$-Poisson space in (2.5) is called in [8] the fusion product of $\left(Y_{j}, \pi_{Y_{j}}, \rho_{j}\right), 1 \leq j \leq n$. 


\section{Ciliated graphs and marked surfaces}

In this section, we review the fusion of marked surfaces and ciliated graphs. Our main references are $[5,7]$.

\subsection{Ciliated graphs and marked surfaces}

A marked surface $(\Sigma, V)$ is a compact Riemann surface, together with a non-empty finite collection of points $V \subset \partial \Sigma$ lying in the boundary of $\Sigma$. A skeleton of a marked surface $(\Sigma, V)$ is a graph $\Gamma$ embedded in $\Sigma$, with set of vertices $V$ and such that $\Sigma$ deformation retracts onto $\Gamma$.

Proposition 3.1 ([7, Section 4]). Any marked surface $(\Sigma, V)$ admits a skeleton, and skeletons of $(\Sigma, V)$ are unique up to isomorphisms and local changes

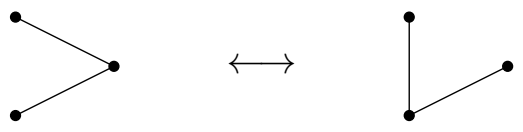

Let $\Gamma$ be a skeleton of a marked surface $(\Sigma, V)$. For every $v \in V$, the orientation of $\Sigma$ induces a linear ordering of the half edges incident to $v$, thus one is led to formulate the following

Definition $3.2([5,7])$. A ciliated graph is a graph in which each vertex is equipped with a linear order of the half edges incident to it.

The name is inspired by the fact that when drawing a ciliated graph, one can specify the linear order of half edges at each vertex by drawing a small "cilium" between the minimal and maximal half edge.
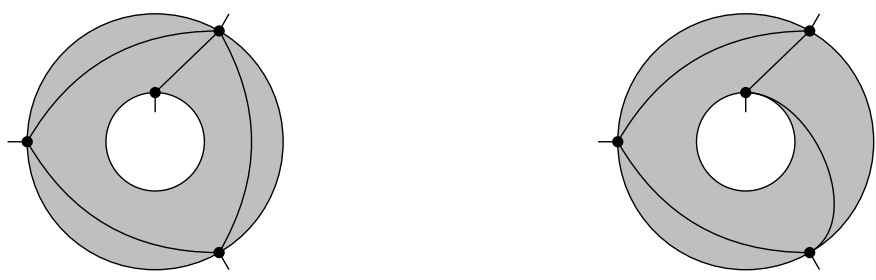

Figure 1. An annulus with four marked points and two non-isomorphic skeletons with their cilium structure.

We introduce further notations in order to discuss ciliated graphs. Let $\Gamma$ be a ciliated graph with set of vertices $V$ and set of edges $\Gamma_{1}$. Denote by $\Gamma_{1 / 2}$ the set of half edges of $\Gamma$, and note that $\Gamma_{1 / 2}$ comes with a natural involution with no fixed points $\alpha \mapsto \check{\alpha}$, mapping a half edge to the opposite half edge, and for $\alpha \in \Gamma_{1 / 2}$ we write $[\alpha, \check{\alpha}]$ for the edge composed of the two half edges $\alpha$ and $\check{\alpha}$. For every $v \in V$, let $\Gamma_{v} \subset \Gamma_{1 / 2}$ be the set of half edges incident to $v$, so that $\Gamma_{1 / 2}=\bigsqcup_{v \in V} \Gamma_{v}$ and $\Gamma_{v}$ is a linearly ordered set for each $v \in V$.

\subsection{Fusion of ciliated graphs and marked surfaces}

We recall now the fusion of marked surfaces and ciliated graphs.

Let $(\Sigma, V)$ be a marked surface. Since $\Sigma$ is oriented, every $v \in V$ defines a piece of arc in $\partial \Sigma$ starting at $v$ and a piece of arc in $\partial \Sigma$ ending at $v$. For a pair $\left(v_{1}, v_{2}\right)$ of distinct elements of $V$, the fusion of $\Sigma$ at $\left(v_{1}, v_{2}\right)$ is the marked surface $\left(\Sigma_{\left(v_{1}, v_{2}\right)}, V_{v_{1}=v_{2}}\right)$ obtained by gluing a piece of arc ending in $v_{1}$ with a piece or arc starting at $v_{2}$, so that $v_{1}$ and $v_{2}$ are identified. The set of marked points $V_{v_{1}=v_{2}}$ on $\Sigma_{\left(v_{1}, v_{2}\right)}$ is then obtained from $V$ by identifying $v_{1}$ and $v_{2}$. 

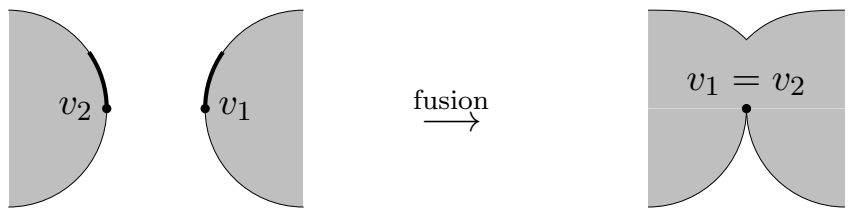

Let $\Gamma$ be a ciliated graph with set of vertices $V$ and edges $\Gamma_{1}$, and let $\left(v_{1}, v_{2}\right)$ be a pair of distinct vertices, with $\Gamma_{v_{1}}=\left\{\alpha_{1}<\cdots<\alpha_{k}\right\}$ and $\Gamma_{v_{2}}=\left\{\alpha_{k+1}<\cdots<\alpha_{l}\right\}$. The fusion of $\Gamma$ at $\left(v_{1}, v_{2}\right)$ is the ciliated graph $\Gamma_{\left(v_{1}, v_{2}\right)}$ obtained by identifying $v_{1}$ and $v_{2}$, and with linear order on the set $\Gamma_{v_{1}=v_{2}}$ of half edges incident to $v_{1}=v_{2}$ given by $\alpha_{1}<\cdots<\alpha_{k}<\alpha_{k+1}<\cdots<\alpha_{l}$.

Note that the fusion of marked surfaces and ciliated graphs are associative operations, but not necessarily commutative. The following lemma is straightforward.

Lemma 3.3. Let $(\Sigma, V)$ be a marked surface with skeleton $\Gamma$, and let $v_{1}, v_{2} \in V$ be distinct points. Then the image of $\Gamma$ under the fusion map $(\Sigma, V) \rightarrow\left(\Sigma_{\left(v_{1}, v_{2}\right)}, V_{v_{1}=v_{2}}\right)$ is isomorphic to $\Gamma_{\left(v_{1}, v_{2}\right)}$, and is a skeleton for $\left(\Sigma_{\left(v_{1}, v_{2}\right)}, V_{v_{1}=v_{2}}\right)$.

Since $\bullet$ is a skeleton for a disk with two marked points, and since every ciliated graph can be obtained by successive fusion of copies of $\longrightarrow$. , every marked surface can be obtained by successive fusion of disks with two marked points. Conversely, a ciliated graph $\Gamma$ with set of edges $V$ is the skeleton of a marked surface $\left(\Sigma_{\Gamma}, V\right)$, well defined up to isomorphism, obtained by fusing marked disks corresponding to the edges of $\Gamma$. Thus the map $\Gamma \mapsto\left(\Sigma_{\Gamma}, V\right)$ gives a bijective correspondence between isomorphism classes of ciliated graphs up to local changes in (3.1) and isomorphism classes of marked surfaces.
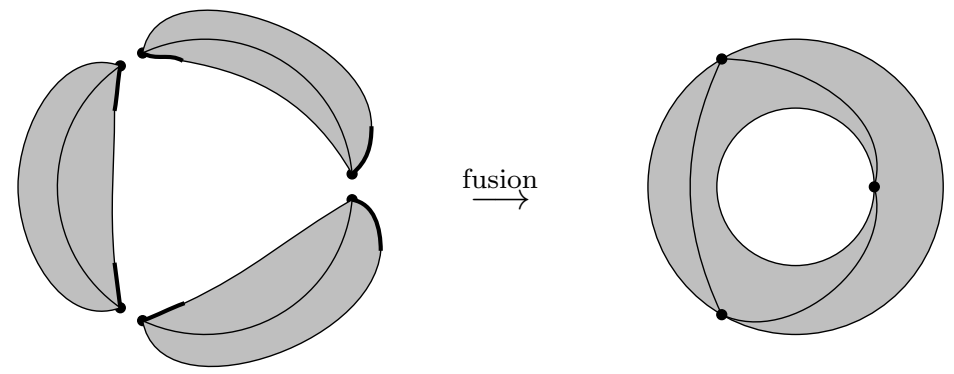

Figure 2. An annulus with three marked points obtained by fusing three disks with two marked points each.

\section{The Fock-Rosly Poisson structure}

In this section, we introduce a Poisson structure, first discovered by Fock and Rosly, on the moduli space of flat connections over a marked surface, which is defined by an action of a Lie algebra and a quasitriangular $r$-matrix.

Throughout Section 4, $G$ is a connected complex Lie group, and $\mathfrak{g}$ is its Lie algebra.

\subsection{The moduli space of flat connections over a marked surface}

For a marked surface $(\Sigma, V)$, let $\Pi_{1}(\Sigma, V)$ be the fundamental groupoid of $\Sigma$ over the set of base points $V$, and consider

$$
\mathcal{A}(\Sigma, V)=\operatorname{Hom}\left(\Pi_{1}(\Sigma, V), G\right),
$$


the moduli space of flat connections on $G$-principal bundles over $\Sigma$ which are trivialized over $V$. The group $G^{V}$ naturally acts on the right on $\mathcal{A}(\Sigma, V)$ by gauge transformations. For $p \in$ $\Pi_{1}(\Sigma, V)$, denote by $\mathrm{ev}_{p}: \mathcal{A}(\Sigma, V) \rightarrow G$ the evaluation at $p$, by $\theta(p), \tau(p) \in V$ the respective source and target of $p$, and if $g \in G^{V}$ and $v \in V$, recall from Section 1.1 that $g_{v}$ is the $v$ 'th component of $g$. The action of $G^{V}$ on $\mathcal{A}(\Sigma, V)$ is then given by

$$
\rho_{V}: \mathcal{A}(\Sigma, V) \times G^{V} \rightarrow \mathcal{A}(\Sigma, V), \quad \operatorname{ev}_{p}\left(\rho_{V}(y, g)\right)=g_{\theta(p)}^{-1} \operatorname{ev}_{p}(y) g_{\tau(p)},
$$

where $g \in G^{V}, y \in \mathcal{A}(\Sigma, V)$, and $p \in \Pi_{1}(\Sigma, V)$. Given a skeleton $\Gamma$ of $(\Sigma, V)$ and an orientation of each edge of $\Gamma, \Pi_{1}(\Sigma, V)$ is then the free groupoid generated by $\Gamma$, and thus one has a natural diffeomorphism

$$
I_{\Gamma}: G^{\Gamma_{1}} \stackrel{\cong}{\longrightarrow} \mathcal{A}(\Sigma, V) .
$$

Now, choose a pair of distinct marked points $\left(v_{1}, v_{2}\right)$. The fusion map $(\Sigma, V) \rightarrow\left(\Sigma_{\left(v_{1}, v_{2}\right)}, V_{v_{1}=v_{2}}\right)$ induces a map

$$
\varphi_{\left(v_{1}, v_{2}\right)}: \mathcal{A}\left(\Sigma_{\left(v_{1}, v_{2}\right)}, V_{v_{1}=v_{2}}\right) \longrightarrow \mathcal{A}(\Sigma, V),
$$

and by choosing a skeleton for $(\Sigma, V)$, one easily sees that $\varphi_{\left(v_{1}, v_{2}\right)}$ is a diffeomorphism. The next lemma is straightforward.

Lemma 4.1. Let $\operatorname{diag}_{\left(v_{1}, v_{2}\right)}: G^{V_{v_{1}=v_{2}}} \rightarrow G^{V}$ be the canonical embedding. Then for any $g \in$ $G^{V_{v_{1}=v_{2}}}$, one has

$$
\varphi_{\left(v_{1}, v_{2}\right)}\left(\rho_{V_{v_{1}=v_{2}}}(y, g)\right)=\rho_{V}\left(\varphi_{v_{1}, v_{2}}(y), \operatorname{diag}_{\left(v_{1}, v_{2}\right)}(g)\right), \quad y \in \mathcal{A}\left(\Sigma_{\left(v_{1}, v_{2}\right)}, V_{v_{1}=v_{2}}\right) .
$$

Let $\Gamma$ be a skeleton of $(\Sigma, V)$, and for any $\gamma \in \Gamma_{1}$, let $\left(D_{\gamma}, V_{\gamma}\right)$ be a disk marked with two points, where $V_{\gamma} \subset \Gamma_{1 / 2}$ consists of the two half edges of $\gamma$, so that $\sqcup_{\gamma \in \Gamma_{1}} V_{\gamma}=\Gamma_{1 / 2}$. As $(\Sigma, V)$ is a fusion of $\left(\sqcup_{\gamma \in \Gamma_{1}} D_{\gamma}, \Gamma_{1 / 2}\right)$, one has a diffeomorphism

$$
\varphi_{\Gamma}: \mathcal{A}(\Sigma, V) \stackrel{\cong}{\longrightarrow} \mathcal{A}\left(\sqcup_{\gamma \in \Gamma_{1}} D_{\gamma}, \Gamma_{1 / 2}\right),
$$

and thus an action of $G^{\Gamma_{1 / 2}}$ on $\mathcal{A}(\Sigma, V)$ via $\varphi_{\Gamma}$. Choosing an orientation for each edge of $\Gamma$ and identifying $\mathcal{A}(\Sigma, V) \cong G^{\Gamma_{1}}$ as in (4.2), the action of $G^{\Gamma_{1 / 2}}$ on $G^{\Gamma_{1}}$ is given by

$$
\begin{aligned}
& \sigma_{\Gamma}: G^{\Gamma_{1}} \times G^{\Gamma_{1 / 2}} \rightarrow G^{\Gamma_{1}}, \\
& \sigma_{\Gamma}(g, h)_{\gamma}=h_{\alpha_{\gamma}}^{-1} g_{\gamma} h_{\check{\alpha}_{\gamma}}, \quad h \in G^{\Gamma_{1 / 2}}, \quad g \in G^{\Gamma_{1}}, \quad \gamma \in \Gamma_{1},
\end{aligned}
$$

where for $\gamma \in \Gamma_{1}, \alpha_{\gamma} \in \Gamma_{1 / 2}$ is the source half edge of $\gamma$.

\subsection{The Fock-Rosly Poisson structure}

Let $(\Sigma, V)$ be a marked surface and $\Gamma$ an oriented skeleton of $(\Sigma, V)$. From now till the end of Section 4, we fix an

$$
s \in\left(S^{2} \mathfrak{g}\right)^{\mathfrak{g}} .
$$

For every $v \in V$, let $\Lambda_{v} \in \wedge^{2} \mathfrak{g}$ be such that $r_{v}=s+\Lambda_{v}$ is a quasitriangular $r$-matrix. Identifying $\mathfrak{g}^{\Gamma v}$ with $\mathfrak{g}^{\left|\Gamma_{v}\right|}$ using the linear order on $\Gamma_{v}$, let $r_{v}^{\left(\varepsilon_{v}, \Gamma_{v}\right)} \in \mathfrak{g}^{\Gamma v} \otimes \mathfrak{g}^{\Gamma v}$ be as in (2.3), where $\varepsilon_{v}: \Gamma_{v} \rightarrow\{-1,1\}$ is defined as

$$
\varepsilon_{v}(\alpha)=\left\{\begin{array}{rl}
1, & \alpha \text { is a source half edge, } \\
-1, & \alpha \text { is an end half edge, }
\end{array} \quad \alpha \in \Gamma_{v} .\right.
$$


Since $\Gamma_{1 / 2}=\bigsqcup_{v \in V} \Gamma_{v}$, one has $\mathfrak{g}^{\Gamma_{1 / 2}}=\bigoplus_{v \in V} \mathfrak{g}^{\Gamma v}$, and recalling our notation in Section 1.1, let

$$
r_{\Gamma}=\sum_{v \in V}\left(r_{v}^{\left(\varepsilon_{v}, \Gamma_{v}\right)}\right)_{v} \in \mathfrak{g}^{\Gamma_{1 / 2}} \otimes \mathfrak{g}^{\Gamma_{1 / 2}}
$$

a quasitriangular structure for the Lie bialgebra

$$
\bigoplus_{v \in V}\left(\mathfrak{g}^{\Gamma_{v}}, \delta_{r_{v}}^{\left(\Gamma_{v}\right)}\right)
$$

where $\delta_{r_{v}}^{\left(\Gamma_{v}\right)}$ is as in (2.4). Using the notational convention in Section 1.1, recall from (4.4) the right Lie algebra action $\sigma_{\Gamma}: \mathfrak{g}^{\Gamma_{1 / 2}} \rightarrow \mathfrak{X}^{1}\left(G^{\Gamma_{1}}\right)$.

Theorem 4.2. The bivector field

$$
\pi_{\Gamma}=\sigma_{\Gamma}\left(r_{\Gamma}\right) \in \mathfrak{X}^{2}\left(G^{\Gamma_{1}}\right)
$$

is a Poisson structure on $G^{\Gamma_{1}}$.

Proof. The symmetric part of $r_{\Gamma}$ is

$$
s_{\Gamma}=\sum_{\gamma \in \Gamma_{1}}(s)_{\alpha_{\gamma}}-(s)_{\check{\alpha}_{\gamma}},
$$

where for $\gamma \in \Gamma_{1}, \alpha_{\gamma} \in \Gamma_{1 / 2}$ is the source half edge of $\gamma$. By (4.4), one has $\sigma_{\Gamma}\left(s_{\Gamma}\right)=0$, thus Theorem 4.2 follows from Proposition 2.1.

Remark 4.3. Let $\Lambda_{\Gamma}$ be the anti-symmetric part of the quasitriangular $r$-matrix $r_{\Gamma}$. The bivector field

$$
\pi_{\Gamma}=\sigma_{\Gamma}\left(\Lambda_{\Gamma}\right)
$$

first appeared in $[4,5]$, where the proof that it is Poisson was left as a computation to be checked. Theorem 4.2 gives a simpler and more conceptual proof that $\pi_{\Gamma}$ is a Poisson structure.

Consider the quasitriangular Lie bialgebra

$$
\left(\mathfrak{g}^{V}, r\right)=\bigoplus_{v \in V}\left(\mathfrak{g}, r_{v}\right), \quad \text { where } \quad r=\sum_{v \in V}\left(r_{v}\right)_{v} \in \mathfrak{g}^{V} \otimes \mathfrak{g}^{V}
$$

For $v \in V$, let $\operatorname{diag}_{v}: \mathfrak{g} \rightarrow \mathfrak{g}^{\Gamma v}, \operatorname{diag}_{v}(x)=\sum_{\gamma \in \Gamma_{v}}(x)_{\gamma}$, for $x \in \mathfrak{g}$, and define the map

$$
\operatorname{diag}_{\Gamma}: \mathfrak{g}^{V} \rightarrow \mathfrak{g}^{\Gamma_{1 / 2}}=\bigoplus_{v \in V} \mathfrak{g}^{\Gamma_{v}}, \quad \operatorname{diag}_{\Gamma}(x)=\sum_{v \in V}\left(\operatorname{diag}_{v}\left(x_{v}\right)\right)_{v}, \quad x \in \mathfrak{g}^{V}
$$

By Theorem 2.2, $\operatorname{diag}_{\Gamma}:\left(\mathfrak{g}^{V}, \delta_{r}\right) \rightarrow\left(\mathfrak{g}^{\Gamma_{1 / 2}}, \delta_{r_{\Gamma}}\right)$ is an embedding of Lie bialgebras, and by Lemma 4.1, one has

$$
\rho_{V}=I_{\Gamma} \circ \sigma_{\Gamma} \circ \operatorname{diag}_{\Gamma}
$$

where $\rho_{V}: \mathfrak{g}^{V} \rightarrow \mathfrak{X}^{1}(\mathcal{A}(\Sigma, V))$ is the derivative of the action by gauge transformations in (4.1). Thus as an immediate consequence, one has

Corollary 4.4. The triple $\left(\mathcal{A}(\Sigma, V), I_{\Gamma}\left(\pi_{\Gamma}\right), \rho_{V}\right)$ is a right $\left(\mathfrak{g}^{V}, r\right)$-Poisson space. 


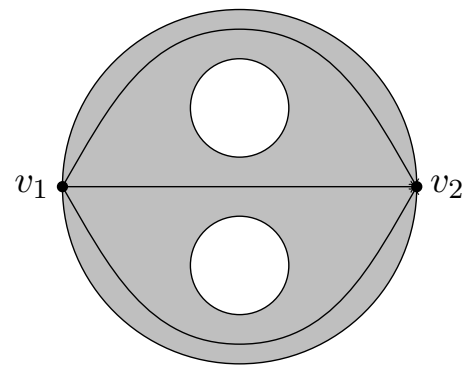

Figure 3. The marked surface $\left(\Sigma_{3}, V\right)$.

Example 4.5. For any integer $n \geq 1$, let $\left(\Sigma_{n}, V\right)$ be a disk with $n-1$ inner disks removed and two marked points $v_{1}, v_{2}$, such as in Fig. 3, and let $\Gamma$ be the skeleton in Fig. 3 with edges oriented from $v_{1}$ to $v_{2}$. Let $r_{i}=s+\Lambda_{i}$, be the $r$-matrix associated to $v_{i}, i=1,2$. The orientation of $\Sigma$ induces an isomorphism $\mathfrak{g}^{\mathrm{\Gamma} v_{i}} \cong \mathfrak{g}^{n}$, and one has

$$
\begin{aligned}
& r_{1}^{\left(\varepsilon_{v_{1}}, \Gamma v_{1}\right)}=(s, \ldots, s)+\left(\Lambda_{1}, \ldots, \Lambda_{1}\right)-\operatorname{Mix}^{n}\left(r_{1}\right)=(s, \ldots, s)+\Lambda_{r_{1}}^{(n)} \\
& r_{2}^{\left(\varepsilon_{v_{2}}, \Gamma v_{2}\right)}=(-s, \ldots,-s)+\left(\Lambda_{2}, \ldots, \Lambda_{2}\right)-\operatorname{Mix}^{n}\left(r_{2}\right)=(-s, \ldots,-s)+\Lambda_{r_{2}}^{(n)},
\end{aligned}
$$

and

$$
I_{\Gamma}\left(\pi_{\Gamma}\right)=\left(\Lambda_{r_{1}}^{(n)}\right)^{R}+\left(\Lambda_{r_{2}}^{(n)}\right)^{L}
$$

In particular, if $\Lambda_{2}=-\Lambda_{1}$, the Poisson structure $I_{\Gamma}\left(\pi_{\Gamma}\right)=\left(\Lambda_{r_{1}}^{(n)}\right)^{R}-\left(\Lambda_{r_{1}}^{(n)}\right)^{L}$ is multiplicative, and the Poisson Lie group

$$
\left(G^{n},\left(\Lambda_{r_{1}}^{(n)}\right)^{R}-\left(\Lambda_{r_{1}}^{(n)}\right)^{L}\right)
$$

is then called a polyuble in [4], and has Lie bialgebra $\left(\mathfrak{g}^{n}, \delta_{r_{1}}^{(n)}\right)$. When $n=2$ and $r_{1}$ is factorizable, that is when $s^{\sharp}: \mathfrak{g}^{*} \rightarrow \mathfrak{g}$ is invertible, $\left(G^{2},\left(\Lambda_{r_{1}}^{(2)}\right)^{R}-\left(\Lambda_{r_{1}}^{(2)}\right)^{L}\right)$ is isomorphic to the double of the Poisson Lie group $\left(G, \Lambda_{1}^{R}-\Lambda_{1}^{L}\right)$ [8]. Thus polyubles are generalizations of doubles of Poisson Lie groups.

\subsection{Independence of choice of skeleton}

Continuing with the setup and notation of Section 4.2, one has a Poisson structure $I_{\Gamma}\left(\pi_{\Gamma}\right)$ on $\mathcal{A}(\Sigma, V)$. The goal of this subsection is to show that $I_{\Gamma}\left(\pi_{\Gamma}\right)$ does not depend on the choice of $\Gamma$, nor on the choice of an orientation of the edges of $\Gamma$. Letting $\Gamma^{\prime}$ be another oriented skeleton of $(\Sigma, V)$, this is equivalent to proving that the map

$$
I_{\Gamma^{\prime}}^{-1} \circ I_{\Gamma}:\left(G^{\Gamma_{1}}, \pi_{\Gamma}\right) \longrightarrow\left(G^{\Gamma_{1}^{\prime}}, \pi_{\Gamma^{\prime}}\right)
$$

is a Poisson isomorphism.

Lemma 4.6. The Poisson structure $I_{\Gamma}\left(\pi_{\Gamma}\right)$ is independent of the orientation of the edges of $\Gamma$.

Proof. One can assume that $\Gamma^{\prime}$ is the same oriented skeleton as $\Gamma$, except for an edge $\gamma \in \Gamma_{1}$, which is given the opposite orientation. The map $I_{\Gamma^{\prime}}^{-1} \circ I_{\Gamma}: G^{\Gamma_{1}} \rightarrow G^{\Gamma_{1}^{\prime}}=G^{\Gamma_{1}}$ is thus the group inversion in the factor $\gamma$, and the identity on all other factors, hence for any $x \in \mathfrak{g}^{\Gamma_{1 / 2}}$, one has

$$
I_{\Gamma^{\prime}}^{-1} \circ I_{\Gamma}\left(\sigma_{\Gamma}(x)\right)=\sigma_{\Gamma^{\prime}}(x),
$$

which implies that $I_{\Gamma^{\prime}}^{-1} \circ I_{\Gamma}\left(\pi_{\Gamma}\right)=\pi_{\Gamma^{\prime}}$. 
Lemma 4.7. Consider the following two oriented skeletons

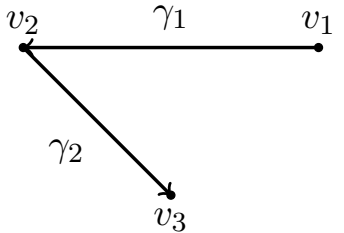

$\Gamma$

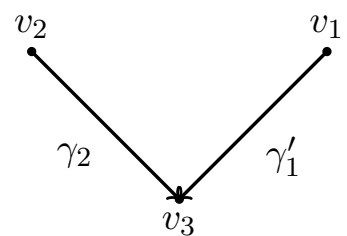

$\Gamma^{\prime}$

of a disk with three marked points. Then the map (4.9) is a Poisson isomorphism.

Proof. Identifying $G^{\Gamma_{1}}$ and $G^{\Gamma_{1}^{\prime}}$ with $G^{2}$ and writing $I=I_{\Gamma^{\prime}}^{-1} \circ I_{\Gamma}$, one has $I\left(g_{1}, g_{2}\right)=\left(g_{1} g_{2}, g_{2}\right)$, $g_{1}, g_{2} \in G$, and

$$
\pi_{\Gamma}=\left(\Lambda_{v_{1}}^{R}, \Lambda_{v_{3}}^{L}\right)+\left(\Lambda_{v_{2}}^{L}, \Lambda_{v_{2}}^{R}\right)+\sum_{i}\left(0, y_{i}^{R}\right) \wedge\left(x_{i}^{L}, 0\right),
$$

where $r_{v_{2}}=\sum_{i} x_{i} \otimes y_{i}$. A direct calculation using $\operatorname{Ad}_{g}(s)=s$ for any $g \in G$, shows that

$$
\begin{aligned}
& \left(\Lambda_{v_{1}}^{R}, 0\right)=I\left(\Lambda_{v_{1}}^{R}, 0\right), \\
& \left(\Lambda_{v_{3}}^{L}, \Lambda_{v_{3}}^{L}\right)-\operatorname{Mix}^{2}\left(\Lambda_{v_{3}}\right)^{L}=I\left(0, \Lambda_{v_{3}}^{L}\right), \\
& \left(0, \Lambda_{v_{2}}^{R}\right)-\operatorname{Mix}^{2}(s)^{L}=I\left(\left(\Lambda_{v_{2}}^{L}, \Lambda_{v_{2}}^{R}\right)+\sum_{i}\left(0, y_{i}^{R}\right) \wedge\left(x_{i}^{L}, 0\right)\right),
\end{aligned}
$$

from which one gets $I\left(\pi_{\Gamma}\right)=\left(\Lambda_{v_{1}}^{R}, \Lambda_{v_{2}}^{R}\right)+\left(\Lambda_{v_{3}}^{L}, \Lambda_{v_{3}}^{L}\right)-\operatorname{Mix}^{2}\left(r_{v_{3}}\right)^{L}=\pi_{\Gamma^{\prime}}$.

We return to the case of a general marked surface $(\Sigma, V)$.

Proposition 4.8. Let $\Gamma, \Gamma^{\prime}$ be oriented skeletons of $(\Sigma, V)$. Then the map (4.9) is a Poisson isomorphism.

Proof. By Lemma 4.6 and Proposition 3.1, one can assume that $\Gamma$ and $\Gamma^{\prime}$ have the following form:

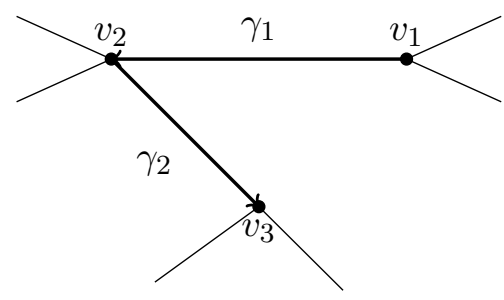

$\Gamma$

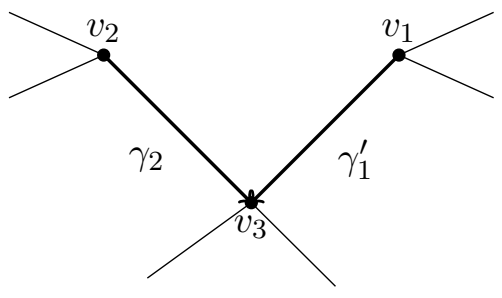

$\Gamma^{\prime}$

Using Lemma 4.7, a straightforward calculation, the details of which are left to the readers, shows that $I_{\Gamma^{\prime}}^{-1} \circ I_{\Gamma}\left(\pi_{\Gamma}\right)=\pi_{\Gamma^{\prime}}$.

Recall the quasitriangular $r$-matrix $r$ on $\mathfrak{g}^{V}$ defined in (4.6) and let

$$
\pi_{r}=I_{\Gamma}\left(\pi_{\Gamma}\right) \in \mathfrak{X}^{2}(\mathcal{A}(\Sigma, V)),
$$

where $\Gamma$ is any oriented skeleton of $(\Sigma, V)$. The following theorem is a consequence of Lemma 4.6, Proposition 4.8, and Lemma 4.4.

Theorem 4.9. The Poisson structure $\pi_{r}$ on $\mathcal{A}(\Sigma, V)$ is independent of the choice of $\Gamma$, and $\left(\mathcal{A}(\Sigma, V), \pi_{r}, \rho_{V}\right)$ is a right $\left(\mathfrak{g}^{V}, r\right)$-Poisson space. 


\subsection{Fusion of Poisson spaces and marked surfaces}

We continue with the setup of Section 4.2. In particular, $(\Sigma, V)$ is a marked surface, for $v \in V$, one has $\Lambda_{v} \in \wedge^{2} \mathfrak{g}$ such that $r_{v}=s+\Lambda_{v}$ is a quasitriangular $r$-matrix, and one considers the quasitriangular $r$-matrix $r \in \mathfrak{g}^{V} \otimes \mathfrak{g}^{V}$ defined in (4.6).

Suppose one has $r_{v_{1}}=r_{v_{2}}$ for two distinct elements $v_{1}, v_{2} \in V$, and consider the fused surface $\left(\Sigma^{\prime}, V^{\prime}\right)=\left(\Sigma_{\left(v_{1}, v_{2}\right)}, V_{v_{1}=v_{2}}\right)$ with Poisson structure $\pi_{r^{\prime}}$, where $r^{\prime} \in \mathfrak{g}^{V^{\prime}} \otimes \mathfrak{g}^{V^{\prime}}$ is defined as in (4.6), and let $v^{\prime} \in V^{\prime}$ be the vertex obtained by fusing $v_{1}$ and $v_{2}$. Recall the fusion of Poisson spaces discussed in Section 2.2.

Theorem 4.10. One has

$$
\operatorname{Fus}_{\left(\mathfrak{g}, r_{v_{1}}\right) \times\left(\mathfrak{g}, r_{v_{2}}\right)}\left(\mathcal{A}(\Sigma, V), \pi_{r}, \rho_{V}\right)=\left(\mathcal{A}\left(\Sigma^{\prime}, V^{\prime}\right), \pi_{r^{\prime}}, \rho_{V^{\prime}}\right) .
$$

Proof. We identify $\mathcal{A}(\Sigma, V)$ and $\mathcal{A}\left(\Sigma^{\prime}, V^{\prime}\right)$ using the natural diffeomorphism $\varphi_{\left(v_{1}, v_{2}\right)}$ in (4.3), and let $\left(\mathcal{A}\left(\Sigma^{\prime}, V^{\prime}\right), \pi^{\prime}, \rho^{\prime}\right)$ be the fusion at $\left(\mathfrak{g}, r_{v_{1}}\right) \times\left(\mathfrak{g}, r_{v_{2}}\right)$ of $\left(\mathcal{A}(\Sigma, V), \pi_{r}, \rho_{V}\right)$. Let $\Gamma$ be an oriented skeleton of $(\Sigma, V)$ and recall from Lemma 3.3 that $\Gamma^{\prime}=\Gamma_{\left(v_{1}, v_{2}\right)}$ is an oriented skeleton of $\left(\Sigma^{\prime}, V^{\prime}\right)$, and that one has a natural identification $\Gamma_{1} \cong \Gamma_{1}^{\prime}$. Recall the map $\operatorname{diag}_{\Gamma}: \mathfrak{g}^{V} \rightarrow \mathfrak{g}^{\Gamma_{1 / 2}}$ defined in (4.7) and consider its restriction $\left.\operatorname{diag}_{\Gamma}\right|_{\mathfrak{g}\left\{v_{1}, v_{2}\right\}}$ to $\mathfrak{g}^{\left\{v_{1}, v_{2}\right\}}$. Using (4.8) and Lemma 2.4, one has

$$
\begin{aligned}
\pi^{\prime} & =\pi_{r}-\left.\rho_{V}\right|_{\mathfrak{g}}\left\{v_{1}, v_{2}\right\} \\
& =I_{\Gamma^{\prime}} \circ \sigma_{\Gamma^{\prime}}\left(r_{\Gamma^{\prime}}\right)=\pi_{r^{\prime}},
\end{aligned}
$$

and by Lemma 4.1 , one has $\rho^{\prime}=\rho_{V^{\prime}}$.

Example 4.11. Let $(\Sigma, V)$ be a disk with two marked points $v_{1}, v_{2}$ and assume that the $r$ matrices $r_{1}$ and $r_{2}$ associated to $v_{1}$ and $v_{2}$ are equal. Let the edge of $\Gamma$ be oriented from $v_{1}$ to $v_{2}$ and identify $\mathcal{A}(\Sigma, V) \cong G$ via $I_{\Gamma}$, so that one has

$$
\pi_{r}=\Lambda^{R}+\Lambda^{L}
$$

where $\Lambda$ is the anti-symmetric part of $r_{1}=r_{2}$.
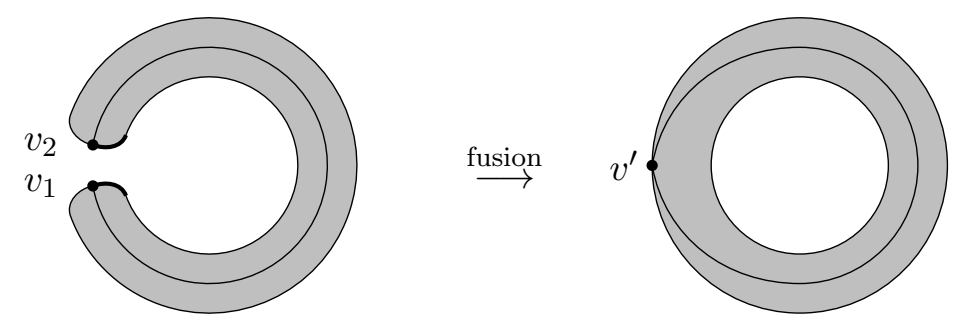

The fused surface $\left(\Sigma^{\prime},\left\{v^{\prime}\right\}\right)$ is an annulus with one marked point, and one has

$$
\begin{aligned}
\pi_{r^{\prime}} & =\Lambda^{R}+\Lambda^{L}-\rho_{V}\left(\operatorname{Mix}^{2}\left(r_{1}\right)\right)=\Lambda^{R}+\Lambda^{L}+\sum_{i} y_{i}^{R} \wedge x_{i}^{L} \\
& =\sum_{i} \frac{1}{2} x_{i}^{R} \wedge y_{i}^{R}+\frac{1}{2} x_{i}^{L} \wedge y_{i}^{L}+y_{i}^{R} \wedge x_{i}^{L},
\end{aligned}
$$

where $r_{1}=r_{2}=\sum_{i} x_{i} \otimes y_{i}$. 


\section{Quasi Poisson geometry}

\subsection{Quasi Poisson spaces and fusion of quasi Poisson spaces}

Let $\mathfrak{g}$ be a Lie algebra, $s \in\left(S^{2} \mathfrak{g}\right)^{\mathfrak{g}}$, and recall the element $\phi_{s} \in\left(\wedge^{3} \mathfrak{g}\right)^{\mathfrak{g}}$ defined in (2.1). Recall from [2] that a right $\left(\mathfrak{g}, \phi_{s}\right)$-quasi Poisson space is a triple $\left(Y, Q_{Y}, \rho\right)$, where $Y$ is a manifold, $\rho: \mathfrak{g} \rightarrow \mathfrak{X}^{1}(Y)$ a right Lie algebra action, and $Q_{Y} \in \mathfrak{X}^{2}(Y)$ is a $\mathfrak{g}$-invariant bivector field on $Y$, such that

$$
\left[Q_{Y}, Q_{Y}\right]=\rho\left(\phi_{s}\right) .
$$

We denote by $\mathcal{Q P}\left(\mathfrak{g}, \phi_{s}\right)$ the category of right $\left(\mathfrak{g}, \phi_{s}\right)$-quasi Poisson spaces, where the morphisms are $\mathfrak{g}$-equivariant smooth maps respecting the quasi Poisson bivector fields, and if $r \in \mathfrak{g} \otimes \mathfrak{g}$ is a quasitriangular $r$-matrix on $\mathfrak{g}$, denote by $\mathcal{P}(\mathfrak{g}, r)$ the category of right $(\mathfrak{g}, r)$-Poisson spaces, where the morphisms are $\mathfrak{g}$-equivariant Poisson maps.

Proposition 5.1 ([1, 6, 8]). Let $\Lambda \in \wedge^{2} \mathfrak{g}$ such that $r=s+\Lambda$ is a quasitriangular $r$-matrix. Then one has an equivalence of categories

$$
\mathcal{P}(\mathfrak{g}, r) \longleftrightarrow \mathcal{Q P}\left(\mathfrak{g}, \phi_{s}\right), \quad\left(Y, \pi_{Y}, \rho\right) \mapsto\left(Y, \pi_{Y}-\rho(\Lambda), \rho\right),
$$

where the functor on the level of morphisms is the identity map.

Proof. Let $\left(Y, \pi_{Y}, \rho\right)$ be a right $(\mathfrak{g}, r)$-Poisson space. Then

$$
\left[\pi_{Y}-\rho(\Lambda), \pi_{Y}-\rho(\Lambda)\right]=-2\left[\rho(\Lambda), \pi_{Y}\right]+\rho([\Lambda, \Lambda])=-\rho([\Lambda, \Lambda])=\rho\left(\phi_{s}\right),
$$

and for $x \in \mathfrak{g}$,

$$
\left[\rho(x), \pi_{Y}-\rho(\Lambda)\right]=\rho\left(\delta_{r}(x)-[x, \Lambda]\right)=0,
$$

hence $\left(Y, \pi_{Y}-\rho(\Lambda), \rho\right)$ is a right $\left(\mathfrak{g}, \phi_{s}\right)$-quasi Poisson space. One similarly checks that if $\left(Y, Q_{Y}, \rho\right)$ is a $\left(\mathfrak{g}, \phi_{s}\right)$-quasi Poisson space, $\left(Y, Q_{Y}+\rho(\Lambda), \rho\right)$ is a right $(\mathfrak{g}, r)$-Poisson space.

\subsection{A canonical quasi Poisson structure on $\mathcal{A}(\Sigma, V)$}

Let $G$ be a connected complex Lie group, $\mathfrak{g}$ its Lie algebra, and let $s \in\left(S^{2} \mathfrak{g}\right)^{\mathfrak{g}}$. Let $(\Sigma, V)$ be a marked surface and for $v \in V$, let $\Lambda_{v} \in \wedge^{2} \mathfrak{g}$ be such that $r_{v}=s+\Lambda_{v}$ is quasitriangular $r$-matrix, and let $r \in \mathfrak{g}^{V} \otimes \mathfrak{g}^{V}$ be as in (4.6). By Proposition 5.1,

$$
\left(\mathcal{A}(\Sigma, V), Q_{s}:=\pi_{r}-\rho_{V}\left(\sum_{v \in V}\left(\Lambda_{v}\right)_{v}\right), \rho_{V}\right)
$$

is a right $\left(\mathfrak{g}, \phi_{s}\right)^{V}$-quasi Poisson space.

Proposition 5.2 (see also [7]). For any oriented skeleton $\Gamma$ of $(\Sigma, V)$, one has

$$
I_{\Gamma}^{-1}\left(Q_{s}\right)=-\sigma_{\Gamma}\left(\sum_{v \in V}\left(\operatorname{Mix}^{\Gamma v}(s)\right)_{v}\right),
$$

where for $v \in V, \operatorname{Mix}^{\Gamma v}(s) \in \wedge^{2}\left(\mathfrak{g}^{\Gamma v}\right) \cong \wedge^{2}\left(\mathfrak{g}^{\left|\Gamma_{v}\right|}\right)$ is the element defined in (2.2) using the linear order of $\Gamma_{v}$. In particular, $Q_{s}$ depends only on $s \in\left(S^{2} \mathfrak{g}\right)^{\mathfrak{g}}$.

Proof. Let $\Lambda_{\Gamma} \in \wedge^{2} \mathfrak{g}^{\Gamma_{1 / 2}}$ be the anti-symmetric part of the quasitriangular $r$-matrix $r_{\Gamma}$ in (4.5) and recall the map $\operatorname{diag}_{\Gamma}: \mathfrak{g}^{V} \rightarrow \mathfrak{g}^{\Gamma_{1 / 2}}$ in (4.7). By Lemma 2.3, one has

$$
I_{\Gamma}^{-1}\left(Q_{s}\right)=\sigma_{\Gamma}\left(\Lambda_{\Gamma}-\operatorname{diag}_{\Gamma}\left(\sum_{v \in V}\left(\Lambda_{v}\right)_{v}\right)\right)=-\sigma_{\Gamma}\left(\sum_{v \in V}\left(\operatorname{Mix}^{\Gamma v}(s)\right)_{v}\right) .
$$


Remark 5.3. Formula (5.1) appears in [7, Section 4], where $Q_{s}$ was defined via fusion of quasi-Poisson spaces.

Note that both $\pi_{r}$ and $Q_{s}$ descend to the same Poisson structure on the moduli space $\mathcal{A}(\Sigma, V) / G^{V}$ of flat $G$-connections over $\Sigma$.

Example 5.4. Let $\left(\Sigma^{\prime},\left\{v^{\prime}\right\}\right)$ be the annulus with one marked point in Example 4.11, and let the edge of its skeleton $\Gamma^{\prime}$ be oriented in the anti-clockwise direction. Identify $\mathcal{A}\left(\Sigma^{\prime},\left\{v^{\prime}\right\}\right) \cong G$ via $I_{\Gamma^{\prime}}$ and $\mathfrak{g}^{\Gamma_{1 / 2}^{\prime}} \cong \mathfrak{g}^{2}$. Writing $r=\sum_{i} x_{i} \otimes y_{i}$, one has $2 s=\sum_{i}\left(x_{i} \otimes y_{i}+y_{i} \otimes x_{i}\right)$, thus

$$
Q_{s}=-\sigma_{\Gamma^{\prime}}\left(\operatorname{Mix}^{2}(s)\right)=\frac{1}{2} \sum_{i} x_{i}^{R} \wedge y_{i}^{L}+y_{i}^{R} \wedge y_{i}^{L} .
$$

Let $\rho$ be the (right) action of $G$ on itself by conjugation. The right $\left(\mathfrak{g}, \phi_{s}\right)$-quasi Poisson space $\left(G, Q_{s}, \rho\right)$ is an example of the Hamiltonian quasi-Poisson spaces which were studied in [2].

\section{Acknowledgements}

The author wishes to thank Jiang-Hua Lu, Marco Gualtieri and Francis Bischoff for their helpful comments. The author also wishes to thank the anonymous referees and editors, whose suggestions helped improve this paper.

\section{References}

[1] Alekseev A., Kosmann-Schwarzbach Y., Manin pairs and moment maps, J. Differential Geom. 56 (2000), 133-165, math.DG/9909176.

[2] Alekseev A., Kosmann-Schwarzbach Y., Meinrenken E., Quasi-Poisson manifolds, Canad. J. Math. 54 (2002), 3-29, math.DG/0006168.

[3] Atiyah M.F., Bott R., The Yang-Mills equations over Riemann surfaces, Philos. Trans. Roy. Soc. London Ser. A 308 (1983), 523-615.

[4] Fock V.V., Rosly A.A., Flat connections and polyubles, Theoret. and Math. Phys. 95 (1993), 228-238.

[5] Fock V.V., Rosly A.A., Poisson structure on moduli of flat connections on Riemann surfaces and the $r$-matrix, in Moscow Seminar in Mathematical Physics, Amer. Math. Soc. Transl. Ser. 2, Vol. 191, Amer. Math. Soc., Providence, RI, 1999, 67-86, math.QA/9802054.

[6] Li-Bland D., Ševera P., Quasi-Hamiltonian groupoids and multiplicative Manin pairs, Int. Math. Res. Not. 2011 (2011), 2295-2350, arXiv:0911.2179.

[7] Li-Bland D., Ševera P., Moduli spaces for quilted surfaces and Poisson structures, Doc. Math. 20 (2015), 1071-1135, arXiv:1212.2097.

[8] Lu J.-H., Mouquin V., Mixed product Poisson structures associated to Poisson Lie groups and Lie bialgebras, Int. Math. Res. Not., to appear, arXiv:1504.06843.

[9] Massuyeau G., Turaev V., Quasi-Poisson structures on representation spaces of surfaces, Int. Math. Res. Not. 2014 (2014), 1-64, arXiv:1205.4898.

[10] Meusburger C., Wise D.K., Hopf algebra gauge theory on a ribbon graph, arXiv:1512.03966. 